\title{
FATORES ASSOCIADOS AO BAIXO PESO AO NASCER NO ESTADO DE GOIÁS
}

\author{
FACTORS ASSOCIATED WITH LOW BIRTH WEIGHT IN GOIÁS STATE \\ FACTORES ASOCIADOS AL BAJO PESO AL NACER EN PROVINCIA DE GOIÁS
}

\section{Ruth Minamisava ${ }^{1}$ Maria Alves Barbosa ${ }^{2}$ Lizete Malagoni ${ }^{3}$ Lourdes Maria Silva Andraus ${ }^{3}$}

RESUMO: Recém-nascidos de baixo peso ao nascer (RNBP) são considerados problemas de saúde pública pela associação com altas taxas de mortalidade e morbidade. Nenhum estudo prévio sobre fatores associados ao RNBP na região central do Brasil foi encontrado na literatura. Assim, este estudo teve como objetivo identificar as prevalências e os fatores associados ao baixo peso ao nascer entre nascidos vivos no Estado de Goiás, Brasil. Foi realizado um estudo transversal tendo como fonte de dados o Sistema de Informações de Nascidos Vivos ligado ao Ministério da Saúde. Examinou-se todos os 92.745 recém-nascidos de gestação única residentes no estado de Goiás, no ano 2000. Usou-se análise de regressão logística para identificar os fatores associados ao RNBP (< 2500 g). Em Goiás, os RNBP apresentaram uma prevalência de $5,96 \%$ e os fatores associados ao baixo peso ao nascer foram: prematuridade, baixa e avançada idade materna, mulheres não casadas, menos de sete consultas de pré-natal, parto não hospitalar e neonatos do sexo feminino. Ações intersetoriais são necessárias para reduzir as desigualdades da saúde materna e infantil.

PALAVRAS-CHAVE: recém-nascido, peso ao nascer, cuidado pré-natal.

ABSTRACT: Low birth weight (LBW) is public health problem, because they are associated with increased risk of morbidity and mortality. No previous studies on factors associated with LBW carried out in central Brazil were found in the literature. The main aims of this study were to determine the prevalence and the factors associated with LBW in children born alive in the State of Goiás, Brazil. A cross-sectional analysis was performed using data from the Live Births Information System from the Brazilian Health Ministry. All 92.745 singleton births in the State of Goiás during the year of 2000 were examined. Logistic regression analysis was used to examine the factors associated with LBW $(<2500 \mathrm{~g})$. In Goiás, the prevalence of LBW was $5.96 \%$ and the most important factors associated with LBW were: prematurity, young and older mothers, unmarried women, mother illiteracy, mothers who had less than seven prenatal care visits, non-hospital delivery, and female infants. Local public health actions are necessary to reduce inequalities in infant and maternal care.

KEYWORDS: newborn, birth weight, prenatal care.

RESUMEN: El bajo peso al nacer (BPN) es considerado un problema de salud pública por la relación con altas tasas de mortalidad y morbilidad. Ningún estudio previo sobre factores asociados al BPN en la región central del Brasil fue encontrado en la literatura. El objetivo de este estudio fue identificar la prevalencia y los factores asociados al bajo peso al nacer entre nacidos vivos en el Estado de Goiás, Brasil. Fue realizado un estudio transversal, siendo su fuente de dados el Sistema de Informaciones de Nacidos Vivos, relacionados con el Ministerio de Salud. Se examinaron a todos los 92.745 recién nacidos de gestación única residentes en el estado de Goiás en el año 2000. Se usó un análisis bi-variado y de regresión logística, para identificar los factores asociados al BPN $(<2500 \mathrm{~g})$. En Goiás, $5,96 \%$ del recién nacidos fueron BPN y los factores asociados identificados fueron: premadurez, poca y avanzada edad materna, mujeres no casadas, menos de siete consultas de prenatal, parto fuera del hospital y neonatos del sexo femenino. Acciones intersectoriales son necesarias para reducir las desigualdades o de la salud materna e infantil.

PALABRAS-CLAVES: recién nacido, peso al nascer, atención prenatal.

\footnotetext{
${ }^{1}$ Enfermeira. Mestre em Ciências da Saúde. Professor Adjunto da Faculdade de Enfermagem da Universidade Federal de Goiás. Rua T-12, 1000 Ap 902-B, Setor Bueno, CEP 74223-180, Goiânia - GO. ruth@fen.ufg.br.

${ }^{2}$ Enfermeira. Doutora em Enfermagem. Professora Titular da Faculdade de Enfermagem da Universidade Federal de Goiás.

${ }^{3}$ Enfermeira. Doutorandas do Programa de Pós Graduação em Ciências da Saúde - Convênio Centro Oeste UnB/UFG/UFMS.

Professora Adjunto da Faculdade de Enfermagem da Universidade Federal de Goiás.
} 


\section{INTRODUÇÃO}

As estimativas globais indicam que o baixo peso ao nascer (BPN) afeta substancialmente a incidência de doenças perinatais, consideradas uma das principais causas de doença e morte infantil (BRIGGS, 2003). Além disso, o BPN ainda é considerado por alguns como o principal problema de saúde pública (PANETH, 1995), um preditor de sobrevivência de recém-nascidos de risco (ROSSO, 2003) e um indicador global de saúde que traduz a eficiência do sistema de saúde local (KABIR, 2002).

Nos países industrializados, a prevalência de BPN gira em torno de $4 \%$ a $6 \%$, enquanto nos países em desenvolvimento é de quase 16\% (KELLEY, 1999). No Brasil, constatou-se a existência de taxas variadas de prevalência de recém-nascidos de baixo peso (RNBP): $10,1 \%$ na cidade do Rio de Janeiro (RJ) (D'ORSI e CARVALHO, 1998), 8,9\% no município de São Paulo (SP) (MONTEIRO et al., 2000a) e 6,6\% em Presidente Prudente (SP) (GOMES e SANTO, 1997).

A maior parte dos RNBP apresenta prematuridade e ou retardo do crescimento intrauterino, sendo que os recém-nascidos pré-termos (RNPT) são associados mais freqüentemente a fatores biológicos maternos e o retardo do crescimento intrauterino, a fatores socioeconômicos (KRAMER, 1987; PANETH, 1995). Não há, entretanto, uma linha divisória clara entre esses fatores, uma vez que os socioeconômicos podem ser mediados pelos biológicos maternos e pelos cuidados pré-natais, assim como a presença de doenças pode afetar a situação socioeconômica. Ademais, as políticas sociais e a descentralização dos serviços de saúde também são fatores relevantes, pois podem reduzir os efeitos da desigualdade da saúde (OPAS, 2000; ROSS et al., 2000; VICTORA et al., 2000).

Outros fatores associados ao BPN foram estudados em diferentes países, dentre os quais estresse, status social, peso e altura dos pais, história de aborto, nascimentos múltiplos, uso de cigarro, álcool, café e drogas e, ainda, o sexo do RN (KRAMER, 1987; BASSO et al., 1997; DAVID e COLLINS, 1997; ROBINSON et al., 2000; BLONDEL et al., 2002).

Estudos brasileiros mostraram associação entre RNBP e parto cesáreo (SILVA et al., 1998; SILVA et al., 2001), nascimento em hospitais privados, história de aborto/natimorto (BETTIOL et al., 2000), RNPT e mães sem escolaridade (MONTEIRO et al., 2000a) e baixa renda (RONDÓ et al., 1997). Já no Rio de Janeiro (RJ), mães com idade entre 15 e 19 anos, nascimento em maternidade pública e nenhuma consulta de pré-natal foram fatores de risco para $\mathrm{RN}$ a termo com BPN (GAMA et al., 2001). Crianças negras apresentaram maior prevalência de BPN em Pelotas (RS) (BARROS et al., 2001).

No estudo de MORAIS NETO \& BARROS (2000), os RNBP constituíram importante grupo de risco para mortalidade infantil nos período neonatal e pós-neonatal em Goiânia (GO), indicando a necessidade de priorizar intervenções que reduzam o impacto desse fator na qualidade de vida das crianças.

Particularmente o estado de Goiás, localizado na Região Centro-Oeste do Brasil, não conta com nenhum estudo que mostre a situação dos recémnascidos com BPN, embora apresente enormes contrastes na distribuição espacial de sua população (GEOGOIÁS, 2002), pequeno número de habitantes na maioria dos seus 242 municípios (IBGE, 2001), grandes desigualdades do índice de desenvolvimento humano municipal (MARTINS, 2003), má distribuição de leitos obstétricos (DATASUS, 2003b) e nenhum médico residente em mais da metade de seus municípios (MARTINS, 2003).

Essas condições aumentam a demanda por informações que subsidiem a tomada de decisões regionais, constituindo importante elemento de pressão para definição de políticas e de estratégias adequadas aos problemas de saúde específicos e justificam a necessidade de investigações de abrangência estadual sobre RNBP e seus fatores de risco.

\section{OBJETIVOS}

Descrever a população de recém-nascidos vivos de gestação única do estado de Goiás a partir dos dados do Sistema de Informações sobre Nascidos Vivos do estado de Goiás referentes ao ano 2000; Identificar e analisar a prevalência e os fatores associados ao baixo peso ao nascer.

\section{MATERIAL E MÉTODOS}

Trata-se de estudo transversal de nascidos vivos de gravidez única residentes no estado de Goiás, Região Centro-Oeste do Brasil, no ano 2000.

A população estudada constituiu-se de todos os registros das Declarações de Nascidos Vivos, perfazendo um universo de 94.637 recém-nascidos vivos. Os dados foram obtidos no Sistema de Informações sobre Nascidos Vivos do Estado de Goiás (SINASC-GO), após processamento final pelo Ministério da Saúde.

O documento fonte do sistema é a Declaração de Nascido Vivo, que é obrigatória (BRASIL, 1973), de obtenção gratuita (BRASIL, 1995) e padronizada em todo o país. Esta declaração contém dados obtidos por meio de registros hospitalares e ou relatados pela mãe/familiar e é a base das informações do Registro Civil. Os registros do SINASC-GO apresentam uma estimativa de sub-registro de apenas $0,48 \%$ (DATASUS, 2003a).

Os dados da população residente foram obtidos do Censo Demográfico - Resultados do Universo ano 2000, realizado pelo Instituto Brasileiro de Geografia e Estatística (2001). A população residente do estado de Goiás, em 2000, era de 5.003.228 habitantes, utilizando-se $1^{\circ}$ de agosto de 2000 como data de referência.

A variável de desfecho foi o baixo peso ao nascer (peso inferior a $2.500 \mathrm{~g}$ ) (UNICEF, 2003). O 
peso ao nascer foi usado como variável contínua, estratificada a cada $500 \mathrm{~g}$ de peso e como variável dicotômica (BPN e não BPN).

As variáveis independentes relativas à mãe foram: idade materna, escolaridade, estado civil, número de filhos, número de consultas de pré-natal, idade gestacional, tipo de parto, local de residência, se parto hospitalar. As relativas ao neonato foram: sexo e cor/raça.

Os dados fornecidos pelo SINASC de Goiás foram transportados para o software Access e exportados para o formato dbf. A seguir, foram importados para os softwares Epi Info (DEAN et al., 2003) e SPSS (NIE et al., 2001) para que fossem efetuados os cálculos apropriados.

Não foram solicitados os registros originais da Declaração de Nascido Vivo (DN) porque o software utilizado pelo Ministério da Saúde, a partir do ano 2000, é programado para solicitar confirmação de dados discrepantes. Embora esse sistema haja aumentado a confiabilidade dos dados comparativamente aos anos anteriores, houve 57 casos com dados inconsistentes em relação à idade materna e o número de filhos (exemplo fictício: mãe de 20 anos de idade com 15 filhos). Nesses casos, optouse pela exclusão do número de filhos e manutenção da idade da mãe quando a somatória do número de filhos vivos e mortos foi maior que o número correspondente à idade materna em anos menos dez.

Para obtenção do número de filhos foram somados os números de filhos vivos e mortos. Quando o número de filhos vivos ou mortos se encontrava em branco ou era ignorado, a variável número de filhos foi registrada como em branco.

Os dados ainda revelaram 951 casos $(1,01 \%)$ de neonatos com os dois índices de Apgar (10 e 5o minuto) iguais a zero, mas nenhum deles com registro de idade gestacional menor que 22 semanas ou peso de nascimento inferior a $500 \mathrm{~g}$. Desses, apenas 16 casos apresentaram idade gestacional entre 22 e 31 semanas de gestação, $11 \mathrm{RN}$ tinham peso entre 500 a $1.500 \mathrm{~g}$ e havia somente um caso de peso ignorado. Nenhum desses registros foi excluído.

A idade gestacional foi estratificada da mesma forma que na DN: menor que 22 semanas, de 22 a 27 semanas, de 28 a 31 semanas, de 32 a 36 semanas, de 37 a 41 semanas e de 42 semanas e mais de gestação. Entretanto, houve 30 registros cuja idade gestacional se encontrava da categoria de 28 a 36 semanas de gestação, não especificada. Como constituíam um baixo número de casos e um intervalo de tempo amplo para a idade gestacional, optou-se por alterar essas caselas para em branco.

Após a limpeza do banco de dados, foram excluídos os casos de gravidez múltipla e ignorada. A seguir, foram realizadas as distribuições de freqüências absoluta e relativa da variável de desfecho e das variáveis de exposição, incluindo a quantidade de registros inválidos (em branco e ignorado) de cada uma delas.

Os estratos das variáveis utilizadas pelo SINASC foram re-agrupados após teste de homogeneidade entre e dentre os grupos. Assim, todos os campos do estrato "menor que 22 semanas de gestação" da variável idade gestacional (36 casos) foram considerados como ignorados. Isso porque pelos testes de Scheffe e de Tukey não foram significantemente diferentes da categoria "32 a 36 semanas de gestação" ( $p=0,721$ e 0,469, respectivamente) e também porque idades gestacionais abaixo do limite de viabilidade estão inclusas nessa categoria.

Para a variável estado civil da mãe, o teste de Tukey indicou a construção de dois estratos em relação ao BNP, mas indicou que os estratos $\mathrm{RN}$ de mães "casada" e de RN de mães em "união consensual" não deveriam ser reunidos em um único estrato (alfa $=5 \%$ ). Por este motivo, essa variável foi categorizada em "casada" e "não casada".

Para as variáveis contínuas - peso ao nascer e idade materna - foram calculadas médias, desvio padrão, medianas e percentis e aplicado o teste de Kolmogorov-Smirnov a fim de se verificar se apresentavam padrão de distribuição de curva normal.

Todos os registros ignorados foram tratados como em branco e, este conjunto recebeu a denominação de registros inválidos. As variáveis com mais de $10 \%$ de registros inválidos foram excluídas das análises uni e multivariada a fim de evitar excesso de casos excluídos, sendo descritas somente as freqüências.

Após a re-construção dos estratos das variáveis, foi realizado cruzamento entre as variáveis peso ao nascer e idade gestacional para identificar a distribuição do peso ao nascer segundo o avanço das semanas gestacionais.

A análise univariada foi realizada para identificar as variáveis associadas ao BPN usando o Statcalc calculator do software Epi Info. Diferenças entre as categorias foram estimadas pelo Odds Ratio (OR) com intervalo de confiança de 95\% (IC95\%). Este cálculo permitiu a seleção das variáveis a serem analisadas por regressão logística.

Somente os fatores que se mostraram significativos na análise univariada com $p<0,20$ foram incluídos no procedimento de regressão logística para identificar os fatores associados ao BPN. Para o procedimento de regressão logística utilizou-se o software SPSS (NIE et al., 2001). A associação entre a exposição e o efeito foi estimada pelo Odds Ratio (OR) com intervalo de confiança de $95 \%$.

O projeto desta pesquisa foi aprovado pelo Comitê de Ética em Pesquisas da Santa Casa de Misericórdia de Goiânia, tal como recomenda a Resolução 196/96 do Conselho Nacional de Saúde (BRASIL, 1996).

\section{RESULTADOS}

A população inicial foi de 94.637 recémnascidos vivos residentes no Estado de Goiás. Considerando a população residente do Estado de Goiás para o ano 2000 de 5.003.228 habitantes (IBGE, 2001), a taxa bruta de natalidade foi de 18,92 nascidos 
vivos por mil habitantes. Excluindo-se os recémnascidos de gravidez múltipla e ignorada, 92.745 casos foram estudados.

A idade materna que variou de 11 a 49 anos, com média de $23,48 \pm 5,58$ anos. Os percentis $25^{\circ}, 50^{\circ}$ e $75^{\circ}$ corresponderam às idades de 19, 23 e 27 anos, respectivamente (gráfico 1 ). Mais de $1 / 4$ dos casos era de mães menores de 20 anos (tabela 1).

Gráfico 1. Distribuição dos nascidos vivos segundo a idade materna.

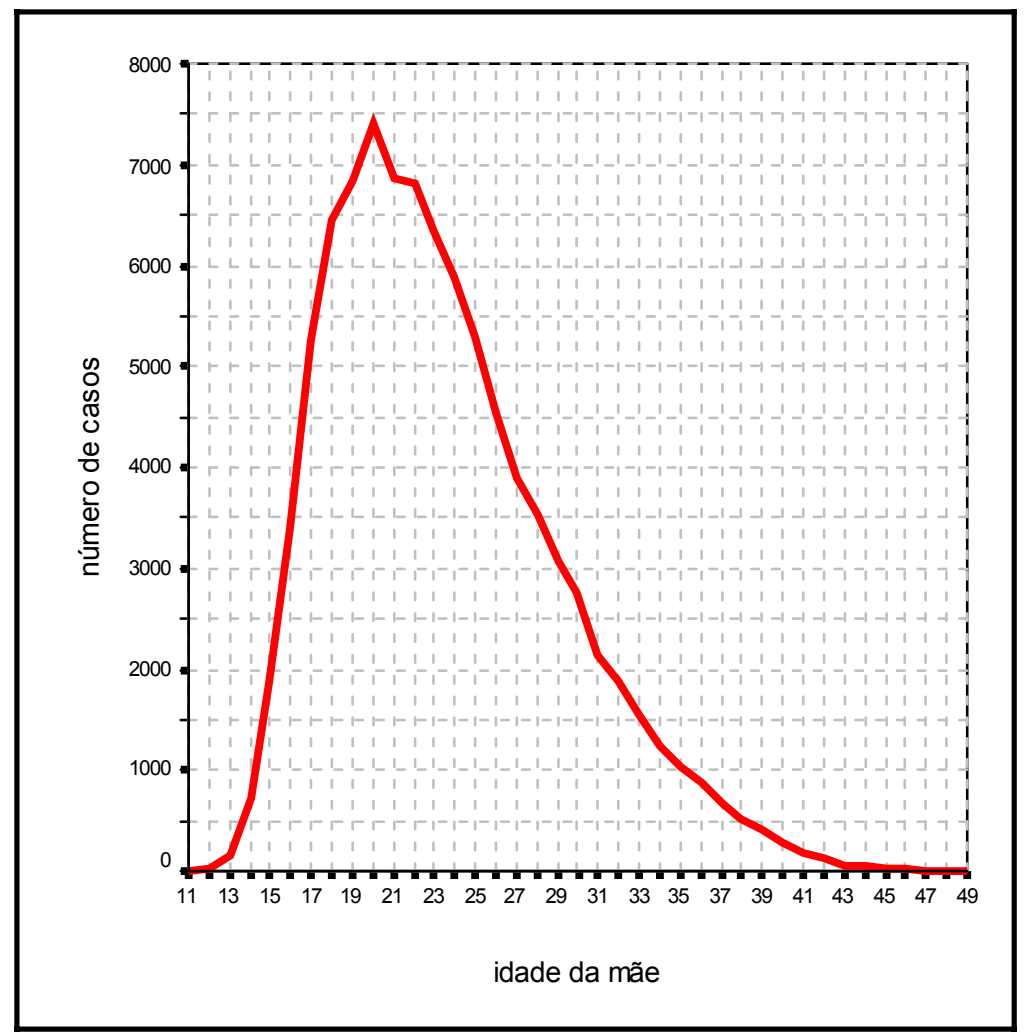

Mães não casadas somaram $51,00 \%$ dos casos e as que nunca freqüentaram a escola, $2,06 \%$. RN a termo totalizaram $92,42 \%$ dos casos e $5,30 \%$ foram de RNPT. O parto cesáreo foi registrado em $44,53 \%$ dos casos. Pouco mais da metade das mães realizaram sete ou mais consultas de pré-natal $(53,28 \%), 2,97 \%$ não fizeram nenhuma consulta. As variáveis número de filhos e raça/cor apresentaram alto porcentual de registros inválidos. Quase a totalidade dos RN nasceu de parto hospitalar, sendo que a maioria dos partos

Tabela 1. Freqüência de nascidos vivos de gravidez única segundo as variáveis de exposição.

\begin{tabular}{lrr}
\hline VARIÁVEIS & $\mathrm{n}$ & \multicolumn{1}{c}{$\%$} \\
\hline Faixa etária da mãe (anos) & & \\
10 a 14 & 896 & 0,97 \\
15 a 19 & 23844 & 25,86 \\
20 a 34 & 63208 & 68,55 \\
35 a 39 & 3528 & 3,83 \\
40 a 49 & 731 & 0,79 \\
registros válidos & 92207 & 99,42 \\
Estado civil da mãe & & \\
Casada & 43972 & 49,00 \\
não casadas & 45767 & 51,00
\end{tabular}

não hospitalares ocorreu no domicílio (dados não mostrados). RN do sexo masculino constituíram $51,45 \%$ da população estudada.

A média do peso ao nascer foi de 3.233,85 \pm $509,16 \mathrm{~g}$, variando entre 310 e $5.700 \mathrm{~g} \mathrm{e}$, os pesos $2.950,3.250$ e $3.550 \mathrm{~g}$ corresponderam aos percentis $25^{\circ}, 50^{\circ}$ e $75^{\circ}$.O padrão de distribuição do peso ao nascer indicou excesso de neonatos com pesos baixos (sk $=-0,523)$. A prevalência de RNBP foi $5,96 \%$ (5507/92391) dos casos (IC95\% 5,81-6,12).

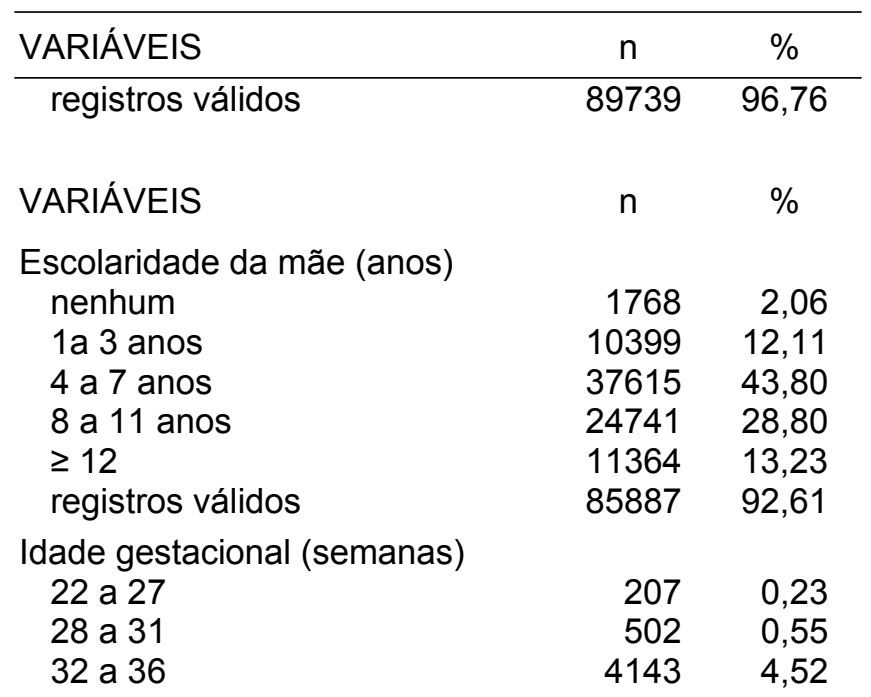




\begin{tabular}{|c|c|c|c|c|c|}
\hline 37 a 41 & 84666 & 92,42 & & & \\
\hline $\begin{array}{l}\geq 42 \\
\text { reaistros válidos }\end{array}$ & $\begin{array}{r}2089 \\
91643\end{array}$ & $\begin{array}{r}2,28 \\
9877\end{array}$ & VARIÁVEIS & $\mathrm{n}$ & $\%$ \\
\hline VARIÁVEIS & $\mathrm{n}$ & $\%$ & $\begin{array}{l}\text { Peso ao nascer }(\mathrm{g}) \\
\quad<500 \\
500-999\end{array}$ & $\begin{array}{r}12 \\
203\end{array}$ & $\begin{array}{l}0,01 \\
0,22\end{array}$ \\
\hline Tipo de parto & & & 1000-1499 & 403 & 0,44 \\
\hline Vaginal & 51383 & 55,47 & 1500-1999 & 888 & 0,96 \\
\hline cesáreo & 41247 & 44,53 & 2000-2499 & 4001 & 4,33 \\
\hline registros válidos & 92630 & 99,88 & 2500-2999 & 19682 & 21,30 \\
\hline Número de consultas de pré-natal & & & $3000-3499$ & 39326 & 42,57 \\
\hline nenhuma & 2629 & 2,97 & $3500-3999$ & 22424 & 24,27 \\
\hline 1 a 3 & 8943 & 10,08 & $4000-4499$ & 4748 & 5,14 \\
\hline 4 a 6 & 29863 & 33,67 & $\geq 4500$ & 704 & 0,76 \\
\hline$\geq 7$ & 47260 & 53,28 & registros válidos & 92391 & 99,62 \\
\hline registros válidos & 88695 & 95,63 & Raça / cor & & \\
\hline Número de filhos (vivos e mortos) & & & branca & 50212 & 63,65 \\
\hline nenhum & 17145 & 36,55 & negra & 1510 & 1,91 \\
\hline 1 & 14047 & 29,95 & amarela & 772 & 0,98 \\
\hline 2 & 8490 & 18,10 & parda & 26064 & 33,04 \\
\hline$\geq 3$ & 7225 & 15,40 & indígena & 328 & 0,42 \\
\hline registros válidos & 46907 & 50,58 & registros válidos & 78886 & 85,06 \\
\hline Parto hospitalar & & & Sexo do RN & & \\
\hline Sim & 92377 & 99,60 & masculino & 47678 & 51,45 \\
\hline Não & 368 & 0,40 & feminino & 44993 & 48,55 \\
\hline registros válidos & 92745 & 100,00 & registros válidos & 92671 & 99,92 \\
\hline
\end{tabular}

Mais da metade dos RNBP $(55,50 \%$, 2.935/5.288) foi composta por neonatos a termo (tabela 2). Dentre os RN não pré-termos, $3,40 \%$ (2.944/86.512) eram de baixo peso. Dos 19.457 recém-nascidos com peso insuficiente (de 2.500 a $2.999 \mathrm{~g}), 91,82 \%$ eram a termo. Dentre os 4.780 RNPT, 49,04\% (2.344/4.780) apresentaram baixo peso ao nascer.

Tabela 2. Peso de nascimento de nascidos vivos segundo a idade gestacional.

\begin{tabular}{|c|c|c|c|c|c|c|c|c|}
\hline \multirow{3}{*}{\multicolumn{2}{|c|}{ PESO AO NASCER }} & \multicolumn{6}{|c|}{ IDADE GESTACIONAL (semanas) } & \multirow{3}{*}{ TOTAL } \\
\hline & & \multicolumn{4}{|c|}{ PRÉ-TERMOS } & \multirow{2}{*}{$\begin{array}{l}\text { TERMOS } \\
37 \text { a } 41 \\
\end{array}$} & \multirow{2}{*}{$\begin{array}{l}\text { Pós-TERMOS } \\
42 \text { e mais }\end{array}$} & \\
\hline & & total $<37$ & 22 a 27 & 28 a 31 & 32 a 36 & & & \\
\hline \multicolumn{2}{|l|}{$<500 \mathrm{~g}$} & 3 & 3 & - & - & - & - & 3 \\
\hline \multicolumn{2}{|c|}{500 a $999 \mathrm{~g}$} & 186 & 115 & 67 & 4 & 2 & - & 188 \\
\hline \multicolumn{2}{|c|}{1000 a $1499 \mathrm{~g}$} & 367 & 63 & 167 & 137 & 8 & - & 375 \\
\hline \multicolumn{2}{|c|}{1500 a $1999 \mathrm{~g}$} & 763 & 6 & 175 & 582 & 50 & 1 & 814 \\
\hline \multicolumn{2}{|c|}{2000 a $2499 \mathrm{~g}$} & 1025 & 3 & 56 & 966 & 2875 & 8 & 3908 \\
\hline \multicolumn{2}{|c|}{2500 a $2999 \mathrm{~g}$} & 1291 & 1 & 11 & 1279 & 17865 & 301 & 19457 \\
\hline \multicolumn{2}{|c|}{3000 a $3499 \mathrm{~g}$} & 965 & 2 & 6 & 957 & 37079 & 899 & 38943 \\
\hline \multicolumn{2}{|c|}{3500 a $3999 \mathrm{~g}$} & 158 & 2 & 2 & 154 & 21474 & 586 & 22218 \\
\hline \multicolumn{2}{|c|}{4000 a $4499 \mathrm{~g}$} & 18 & - & - & 18 & 4454 & 226 & 4698 \\
\hline \multicolumn{2}{|c|}{4500 a $4999 \mathrm{~g}$} & 4 & - & - & 4 & 609 & 23 & 636 \\
\hline \multicolumn{2}{|c|}{5000 a $5499 \mathrm{~g}$} & - & - & - & - & 28 & 19 & 47 \\
\hline \multicolumn{2}{|c|}{5500 a $5999 \mathrm{~g}$} & - & - & - & - & 3 & 2 & 5 \\
\hline \multirow[t]{2}{*}{ TOTAL } & $\mathrm{n}$ & 4780 & 195 & 484 & 4101 & 84447 & 2065 & 91292 \\
\hline & $\%$ & 5,24 & 0,21 & 0,53 & 4,49 & 92,5 & 2,26 & 100,00 \\
\hline \multirow[t]{2}{*}{ BPN * } & $\mathrm{n}$ & 2344 & 190 & 465 & 1689 & 2935 & 9 & 5288 \\
\hline & $\%$ & 49,04 & 97,44 & 96,07 & 41,19 & 3,48 & 0,44 & 5,79 \\
\hline MBP ** & $\%$ & 11,63 & 92,82 & 48,35 & 3,44 & 0,01 & 0,00 & 0,62 \\
\hline
\end{tabular}

$\begin{array}{ll}* & \text { BPN }=\text { baixo peso ao nascer }(<2500 \mathrm{~g}) \\ * & \text { MBP }=\text { muito baixo peso ao nascer }(<1500 \mathrm{~g})\end{array}$ 
Em análise bivariada, todas as variáveis foram associadas com o baixo peso ao nascer, sendo que o parto cesáreo teve efeito protetor (tabela 3 ).

Os fatores associados à RNBP após ajustamento foram mães com RNPT, menores de 20 e maiores de 34 anos de idade, não casadas, com nenhum ano de escolaridade, que realizaram menos que sete consultas de pré-natal, nasceram em ambiente não hospitalar e RN do sexo feminino. Os maiores riscos foram para mães com parto prematuro, que fizeram de nenhuma a três consultas de pré-natal e para os extremos das faixas etárias. Parto cesáreo foi fator protetor para o BPN.

As proporções de RNPT do sexo masculino e do sexo feminino foram de 5,30 e $5,28 \%$, respectivamente, sendo que a razão de sexos foi 0,5155 (2497/4844) (dados não mostrados).

Tabela 3. Distribuição e Odds Ratio de recém-nascidos de baixo peso de gravidez única segundo características dos nascidos vivos.

\begin{tabular}{|c|c|c|c|c|c|c|}
\hline CARACTERÍSTICAS & $\mathbf{n}$ & BPN (\%) & OR bruto & IC (95\%) & $\begin{array}{l}\text { OR ajust. } \\
(\mathrm{n}=80899)\end{array}$ & IC (95\%) \\
\hline Pré-termo & 91607 & & & & & \\
\hline não & 86755 & 3,40 & 1,00 & & 1,00 & \\
\hline $\operatorname{sim}$ & 4852 & 49,04 & 27,31 & $25,51-29,24$ & $27,85^{*}$ & $25,87-29,99$ \\
\hline Idade materna (anos) & 91866 & & & & & \\
\hline 20 a 34 & 63000 & 5,18 & 1,00 & & 1,00 & \\
\hline 10 a 14 & 891 & 12,68 & 2,66 & $2,16-3,26$ & $1,70^{\dagger}$ & $1,30-2,22$ \\
\hline 15 a 19 & 23746 & 7,34 & 1,45 & $1,37-1,54$ & $1,20^{*}$ & $1,11-1,29$ \\
\hline 35 a 39 & 720 & 10,83 & 2,22 & $1,74-2,82$ & $1,58^{*}$ & $1,35-1,84$ \\
\hline 40 a 49 & 3509 & 8,09 & 1,61 & $1,42-1,83$ & $1,87^{\dagger}$ & $1,37-2,54$ \\
\hline Estado civil & 89413 & & & & & \\
\hline casada & 43851 & 4,93 & 1,00 & & 1,00 & \\
\hline não casada & 45562 & 6,93 & 1,44 & $1,36-1,52$ & $1,23^{*}$ & $1,15-1,32$ \\
\hline Escolaridade da mãe & 85573 & & & & & \\
\hline 12 anos e mais & 11337 & 4,61 & 1,00 & & 1,00 & \\
\hline 8 a 11 anos & 24665 & 5,47 & 1,20 & $1,08-1,33$ & $1,01^{\ddagger}$ & $0,89-1,14$ \\
\hline 4 a 7 anos & 37488 & 6,31 & 1,39 & $1,26-1,54$ & $1,07^{\ddagger}$ & $0,95-1,20$ \\
\hline 1 a 3 anos & 10352 & 6,72 & 1,49 & $1,32-1,68$ & $1,02^{\ddagger}$ & $0,88-1,17$ \\
\hline nenhum & 1731 & 9,59 & 2,19 & $1,82-2,64$ & $1,37^{\S}$ & $1,10-1,72$ \\
\hline Consultas de pré-natal & 88373 & & & & & \\
\hline 7 e mais & 47148 & 4,43 & 1,00 & & 1,00 & \\
\hline 4 a 6 & 29757 & 6,62 & 1,53 & $1,44-1,63$ & $1,17^{\dagger}$ & $1,08-1,26$ \\
\hline 1 a 3 & 8881 & 9,51 & 2,27 & $2,09-2,47$ & $1,61^{*}$ & $1,45-1,79$ \\
\hline nenhuma & 2587 & 11,67 & 2,85 & $2,51-3,25$ & $2,06^{*}$ & $1,75-2,43$ \\
\hline Parto hospitalar & 92391 & & & & & \\
\hline $\operatorname{sim}$ & 92094 & 5,94 & 1,00 & & 1,00 & \\
\hline não & 297 & 11,11 & 2,06 & $1,40-3,00$ & $1,61^{* *}$ & $1,03-2,53$ \\
\hline Tipo de parto & 92279 & & & & & \\
\hline vaginal & 51154 & 6,98 & 1,00 & & 1,00 & \\
\hline cesáreo & 41125 & 4,70 & 0,66 & $0,62-0,70$ & $0,76^{*}$ & $0,70-0,81$ \\
\hline Sexo do RN & 92318 & & & & & \\
\hline masculino & 47501 & 5,49 & 1,00 & & 1,00 & \\
\hline feminino & 44817 & 6,46 & 1,19 & $1,13-1,26$ & $1,27^{*}$ & $1,18-1,35$ \\
\hline $\begin{array}{ll}* & p<0,0001 \\
\dagger & p<0,001 \\
\ddagger & p>0,05-\text { não significativo } \\
\$ & p<0,01 \\
* * & p<0,05\end{array}$ & & & & & & \\
\hline
\end{tabular}

\section{DISCUSSÃO}

Os resultados deste trabalho mostraram altas proporções de mães com idade menor que 20 anos, de parto cesáreo e de baixa cobertura de assistência pré-natal.
A porcentagem de recém-nascidos de gestação múltipla foi de 1,84\% (IC95\% 1,76-1,93), mostrando-se próxima à do Rio Grande do Sul no ano de 1999 $(1,93 \%$, IC $95 \% 1,87-2,00)$, porém maior que a da Bahia $(1,69 \%$, IC95\% 1,64-1,75) e a de Pernambuco $(1,67 \%$, IC95\% 1,61-1,74) (FUNASA, 2003). 
Blondel et al. (2002) encontraram prevalências maiores de gravidez múltipla em países desenvolvidos, como EUA $(2,86 \%)$, Inglaterra e País de Gales $(2,87 \%)$, França $(2,94 \%)$ e Canadá $(2,41 \%)$, sugerindo que elevadas proporções de gravidez múltipla podem ser decorrentes do aumento da idade materna e do tratamento da subfertilidade.

\section{Taxa bruta de natalidade}

A taxa bruta de natalidade brasileira, segundo o Ministério da Saúde (2002), é de 20,69 nascidos vivos por mil habitantes, e a menor taxa é a do Estado do Rio Grande do Sul $(17,35 \%$ \%o). Nesta investigação encontrou-se uma taxa bruta de natalidade em Goiás de $18,92 \%$ o.

Esses resultados são condizentes com os dados oficiais do Brasil que indicam as populações das Regiões Centro-Oeste, Sudeste e Sul do país como aquelas que, historicamente, têm maiores índices de desenvolvimento, mais acesso aos serviços básicos de educação, saneamento e saúde e, nos últimos anos, maior redução da taxa de natalidade (IBGE, 2003c; MINISTÉRIO DA SAÚDE, sd.).

A tendência de melhoria dos indicadores de desenvolvimento ficou mais evidente após a divulgação do Censo 2000, que registrou outras mudanças entre 1995 e 1999 ligadas à condição de saúde da Região Centro-Oeste: redução do tamanho das famílias, aumento da instrução das mulheres, redução da concentração de renda e aumento da proporção de moradias urbanas com iluminação elétrica (PNAD, 1999).

A taxa bruta de natalidade do estado de Goiás foi próxima à de países em desenvolvimento como o Chile $(18,5 \%$ oo em 2001 (SZOT MEZA, 2003), mas inferior à encontrada em países mais desenvolvidos como Portugal - $12,0 \%$, (PORTUGAL, 2003) e Estados Unidos - $14,4 \%$ oo (VENTURA et al., 2003).

\section{Idade materna}

Houve uma proporção mais elevada $(26,83 \%$, IC95\% 26,55-27,12) de recém-nascidos de mães menores de 20 anos de idade, quando comparada aos $17,5 \%$ de Ribeirão Preto (SP) (RIBEIRO et al., 2000), aos $17,7 \%$ de outros municípios do Estado de São Paulo (COSTA e GOTLIEB, 1998) e aos $17,2 \%$ de Pelotas (RS) (BARROS et al., 2001).

Pode ter ocorrido aumento na proporção de mães adolescentes ao longo do tempo, como encontrado em Ribeirão Preto (SP) por Ribeiro et al. (2000), em razão da tendência brasileira de redução na idade da primeira relação sexual (BERQUÓ et al., 1999), associada a relações sexuais não protegidas e a gestações não planejadas e ou indesejadas nessa faixa etária (OLINTO e GALVÃO, 1999; BROWN et al., 2001). Além disso, variações culturais relacionadas à sexualidade e à maternidade dentro de um país de grandes dimensões como o Brasil também podem ter um papel importante na alta prevalência de filhos de mães adolescentes.

Um estudo de distribuição espacial da proporção de mães adolescentes por bairros da cidade do Rio de
Janeiro apontou valores mais elevados em áreas com menor infra-estrutura (D'ORSI e CARVALHO, 1998). Mesmo em países desenvolvidos como a Escócia, o aumento da proporção de gestação na adolescência dos últimos anos ocorreu somente para as jovens que residiam em áreas mais carentes (MCLEOD, 2001).

Isso também poderia explicar os resultados do presente estudo, uma vez que a Região Centro-Oeste, apesar dos progressos citados, ainda apresenta uma das maiores concentrações de renda do país. Em Goiás, entre 2001 e 2002, somente $74,9 \%$ dos domicílios permanentes eram atendidos por rede geral de abastecimento de água e $34,6 \%$ possuíam instalação sanitária ligada à rede coletora de esgoto ou à fossa séptica (IBGE, 2003b) e, no ano 2000, 31,40\% das famílias residentes em domicílios particulares tinham rendimento mensal de até dois salários mínimos (IBGE, 2003a).

Entre os estados brasileiros, a proporção de RN de mães com idade acima de 34 anos em Goiás $(4,62 \%$, IC95\% 4,49-4,76), em 2000, foi superior, somente à do estado de Rondônia $(4,21 \%$ IC95\% $3,99-4,43)$. Foi semelhante à dos estados do Mato Grosso $(4,62 \%$ IC95\% 4,44-4,81) e Tocantins $(4,59 \%$, IC95\% 4,34-4,85). Nos outros estados do Brasil, a proporção de RN de mães com idade avançada foi maior, variando de $13,48 \%$ (IC95\% 13,32-13,64) no Rio Grande do Sul a 5,32\% (IC95\% 5,18-5,46) no Maranhão (DATASUS, 2003a).

A proporção de RN de mães com idade superior a 34 anos do Estado de Goiás foi mais baixa que a encontrada por Bortman (1998), na província argentina de Neuquém, 7,08\% (IC95\% 6,72-7,45).

É provável que o conjunto de mães brasileiras com idade avançada represente não apenas primíparas idosas, mas principalmente grandes multíparas, visto que, em países desenvolvidos como os EUA, havia somente $2,21 \%$ de mães nessa faixa etária em 1999 (VENTURA et al., 2001).

Resumidamente, nossos resultados indicam que mais de $1 / 4$ das mães eram de baixa idade, fato possivelmente vinculado às precárias condições sociais e econômicas. Essa situação é preocupante para a saúde pública e deve ser foco de políticas específicas de prevenção e assistência a essa clientela.

\section{Estado civil}

Os resultados sobre o estado civil das mães de Goiás $(34,23 \%$ de solteiras, separadas judicialmente ou viúvas) se revelaram discordantes dos descritos para o estado pelo Instituto Brasileiro de Geografia e Estatística (IBGE, 2003c), que reportou 17,1\% e 17,8\% de famílias compostas por mulheres sem cônjuge e com filhos, em 1999 e 2001, respectivamente.

Uma possível explicação para este fenômeno, observada nas maternidades da capital goiana, está na abordagem da mãe durante o preenchimento da Declaração de Nascido Vivo. Como no formulário há o título estado civil, por vezes a pergunta é feita somente com esse termo, dando margem à compreensão de que as uniões consensuais não estão incluídas. 
Adicionalmente, as mulheres separadas não judicialmente e as que se separaram após união consensual não encontram uma opção clara para responder e podem optar pela alternativa solteira. De modo análogo, quando há união consensual após a viuvez, pode ocorrer escolha pela última.

\section{Número de consultas de pré-natal}

Para cerca de $47 \%$ dos $\mathrm{RN}$, as mães fizeram menos que sete consultas de pré-natal e em 2,97\% dos casos nenhuma consulta foi registrada. Considerando a realização de sete ou mais consultas pré-natais como um indicador de acesso adequado ao serviço de saúde, a baixa cobertura pode refletir a dificuldade no acesso aos serviços de saúde e maior probabilidade de riscos à saúde da mãe e do neonato.

Puccini et al. (2003) encontraram, em Embu (SP), uma proporção de $66,7 \%$ (IC95\% 55,8-77,5) de RN de mães que realizaram seis ou mais consultas de pré-natal, considerando esse um indicador de acesso. Já o estudo de Monteiro et al. (2000b), na cidade de São Paulo (SP), verificou que $88,6 \%$ das mães realizaram cinco ou mais consultas. Essas investigações constataram nítida desvantagem para as faixas inferiores de renda, revelando que grupos mais vulneráveis receberam pior assistência. Sobre este aspecto, Victora et al. (2000) postulam que novas intervenções e programas de saúde pública atingem inicialmente as populações com elevado nível socioeconômico e, só mais tardiamente, os mais pobres.

A cobertura do sistema de saúde brasileiro apresenta variações: em Ribeirão Preto (SP) 9,0\% dos $\mathrm{RN}$ eram de mães que realizaram menos que quatro consultas (SILVA et al., 1998) e, em Feira de Santana (BA), $14,2 \%$ de mães que não realizaram o pré-natal (COSTA et al., 2001).

Pretende-se ampla cobertura dos serviços de pré-natal, pois seus objetivos são prover cuidados médicos, nutricionais e educacionais e identificar mães com risco de parto antecipado ou baixa taxa de crescimento fetal. Esse trabalho tem como finalidade reduzir o baixo peso ao nascer e evitar outros resultados indesejados (ALEXANDER e KORENBROT, 1995), além de melhorar as condições do parto, do puerpério e dos cuidados para com o bebê. Consideramos que o monitoramento desse serviço pode avaliar as desigualdades e que, para evitar a manutenção dessa situação, é necessário definir políticas apropriadas para melhorar a equidade e o acesso à saúde.

\section{Tipo de parto}

A alta prevalência de partos cesáreos é registrada em muitas regiões, mas taxas maiores ou iguais às do estado de Goiás (44,53\%) são raras. Embora não haja consenso sobre qual seria a taxa recomendável de partos cirúrgicos, é difícil crer que quase $45 \%$ das mulheres não apresentem condições de ter seus filhos pela via vaginal, o que fortalece a suposição de que há outras razões que não as indicações obstétricas.
No Brasil, as taxas de partos cirúrgicos variam muito, mas a taxa nacional de 32,4\% em 1995 foi reduzida para 25,2\% em 2001 (MINISTÉRIO DA SAÚDE, sd.). A proporção de partos cesáreos no Brasil variou de $14,51 \%$ no Amapá a $49,33 \%$ no Rio de Janeiro (DATASUS, 2002).

Taxas de cesarianas também variam muito entre os países. Na América do Sul, Belizán et al. (1999) registraram proporções de $15,8 \%$ na Bolívia e de $40,0 \%$ no Chile, associando altas prevalências de partos cesáreos com melhores condições econômicas. Entretanto, nos Estados Unidos a taxa foi de $22 \%$ em 1999 (VENTURA et al., 2001) e, na Europa, verificouse, em Dublin, redução nas proporções de $6,1 \%$ a $4,2 \%$ no período de 1997 a 2000 (EOGAN et al., 2003 ); enquanto na Inglaterra, elas aumentaram de $4 \%$ para $16 \%$ e $20 \%$ em 1970, 1995 e 1999, respectivamente (DOBSON, 2001).

Os trabalhos que analisam esta prática obstétrica descrevem alguns fatores relacionados e ou explicações para as altas taxas de parto cesáreo: conveniência de uma intervenção programada; formação médica deficiente para acompanhamento do parto vaginal; "pressão" por parte das parturientes; compreensão do procedimento cirúrgico como melhor assistência; oportunidade de esterilização durante uma cesárea eletiva; crença de que é um procedimento seguro e de tecnologia avançada; medo da dor e da perda da elasticidade vaginal por parte das mulheres (BELIZÁN et al., 1999; GOMES et al., 1999; HALL e BEWLEY, 1999; LANCET, 2000; WAGNER, 2000; POTTER et al., 2001; BÉHAGUE et al., 2002).

Vale ressaltar ainda o trabalho de Hotimsky et al. (2002), que analisou os motivos que levaram mães a desejar parto cesáreo no Rio de Janeiro (RJ). As autoras indicaram que mais que o medo da dor do parto, as mulheres temiam as reações dos profissionais às suas queixas. Elas preferiam o parto vaginal e temiam a cesárea, pelos riscos a ela associados. Apesar disso, elas referiram que a qualidade da atenção, particularmente na sala de préparto, poderia ser melhor se o parto fosse cesáreo.

Cesáreas desnecessárias são potencialmente iatrogênicas e suscitam questionamentos políticos, econômicos, técnicos e éticos sobre essa prática. Para Rattner (1996), o parto cesáreo, além de um procedimento médico, parece ter adquirido caráter de atividade lucrativa, transformando-se em um bem de consumo.

\section{Idade gestacional}

A baixa prevalência de RNBP em Goiás se justifica, pelo menos parcialmente, pela menor taxa de RNPT. Os RNPT representaram 5,30\% (IC95\% 5,15$5,44)$ dos neonatos residentes no estado de Goiás. Essa proporção foi menor que a do estado de São Paulo - 7,3\% (KILSZTAJN et al., 2003) e que as das cidades de Pelotas (RS) - 8,0\% (HORTA et al., 1997) e de Ribeirão Preto (RS) - 14,8\% (SILVA et al., 1998).

A Estônia também apresentou prevalência de $5,3 \%$, em uma fase de transição para a democracia e a economia de mercado, após um longo período de 
acentuada deteriorização da situação econômica e da expectativa de vida (KOUPILOVA et al., 2000). A França apresentou proporção menor de RNPT que as encontradas em nossos resultados $(4,6 \%)$ (BLONDEL et al., 2002). Já no Canadá (BLONDEL et al., 2002) e nos EUA (VENTURA et al., 2001), as prevalências foram mais altas que em Goiás $(5,9 \%$ e $11,8 \%$, respectivamente).

Apesar do julgamento clínico para estimar o peso fetal ser considerado suficientemente acurado (MEHDIZADEH et al., 2000; OTT, 2002), não se pode descartar que diferentes critérios de classificação da idade gestacional, aliadas à elevada quantidade de pequenos municípios (IBGE, 2001), muitos dos quais desprovidos de profissionais de saúde qualificados, podem subestimar a prevalência de RNPT.

\section{Peso ao nascer}

A média do peso ao nascer neste estudo foi de $3.233,85 \pm 509,16 \mathrm{~g}$. Um estudo realizado na cidade de São Paulo em 1998, incluindo gravidez múltipla, encontrou média de $3.157 \mathrm{~g}$ (MONTEIRO et al., 2000a). Médias de peso ao nascer abaixo de $3.400 \mathrm{~g}$ foram atribuídas à precária qualidade de vida de populações cuja renda é, freqüentemente, tão baixa, que a maior parte dela é gasta com alimentos, o trabalho é fisicamente árduo e as práticas de saúde pública são insuficientes para os padrões modernos (BAKKETEIG et al., 1998).

Este estudo revelou uma prevalência de $0,67 \%$ de RN de muito baixo peso ao nascer (menor que $1.500 \mathrm{~g}$ ). Os dados oficiais brasileiros, que incluem RN de gestações múltiplas, mostraram prevalência de $1,15 \%$ no estado de Minas Gerais e de $1,03 \%$ no Paraná, no mesmo ano (DATASUS, 2003a).

Deve-se considerar, no entanto, a possibilidade o maior número de registro em branco ou ignorado para RN com peso muito baixo (HECK et al., 1999) e também de não emissão da DN para os RN de muito baixo peso, principalmente aqueles julgados abaixo do limite de viabilidade (ALMEIDA e MELLO JORGE, 1996).

A proporção de RN residentes em Goiás com peso entre $2.500 \mathrm{~g}$ a $2.999 \mathrm{~g}$ foi de $21,30 \%$. Dados sobre os estados brasileiros incluindo gestações múltiplas mostraram proporções de $24,60 \%$ em São Paulo, 22,92\% no Paraná, $20,89 \%$ em Santa Catarina e $20,69 \%$ no Espírito Santo (DATASUS, 2003a).

\section{Prevalência - RNBP}

As menores prevalências mundiais de RNBP são de 3\% (Albânia) e 4\% (Suécia). As maiores são de $42 \%$ na Mauritânia e 30\% na Índia e Bangladesh. São exemplos de prevalência entre $6 \%$ e $7 \%$, no período entre 1998 e 2002, os países: França, Alemanha, Itália, Áustria, Suíça, Irlanda, Canadá, Cuba, Costa Rica, Argentina, Venezuela, Líbano, Kuwait, Croácia, República Tcheca, Rússia, China, Nova Zelândia, Austrália (UNICEF, 2003).

No Brasil, a prevalência de BPN, incluídos os de gestação múltipla, foi de $7,70 \%$ no ano 2000 e os valores por região do país foram: Sudeste $-8,62 \%$, Sul
- 8,09\%, Centro-Oeste - 7,05\%, Nordeste - 6,79\% e Norte - 6,31\% (DATASUS, 2003a). Segundo a mesma fonte de dados, os estados de Sergipe e São Paulo apresentaram, respectivamente, prevalências de $6,80 \%$ e $8,67 \%$.

A prevalência de RNBP relativamente baixa do Estado de Goiás $(5,96 \%)$ que Ihe asseguraria uma posição razoavelmente confortável pode ser traduzida como uma informação até positiva. Contudo, pode refletir a zona cinza existente entre natimortos e nascidos vivos com extremo baixo peso. Uma parcela de nascidos vivos pode ser considerada como natimorta por apresentar Índice de Apgar zero e não ser ressuscitada em função da baixa idade gestacional e ou do extremo baixo peso.

O conceito de limite de viabilidade do RN, baseado em definições desatualizadas e até mesmo na experiência dos profissionais de saúde, pode se traduzir na variabilidade dos padrões de conduta da prática médica, particularmente na decisão por realizar cuidados extraordinários.

Sobre este aspecto, um estudo conduzido no Alabama (EUA) mostrou que a deficiência de conhecimento sobre as possibilidades de sobrevivência de RN pré-termos de muito baixo peso pode predispor pediatras a realizar menos tentativas de manutenção da vida (HAYWOOD et al., 1998).

O processo de decisão de realizar medidas extraordinárias de ressuscitação é complexo e envolve o dilema ético de prolongamento da vida ante a incerteza do prognóstico. O profissional de saúde pode justificar a execução de cuidados extraordinários pelos aspectos legais envolvidos e pelo desejo dos pais, correndo o risco de ser criticado pelo zelo extremado. Pode também considerar que a morte é inevitável, portanto o sofrimento e a dor da família e os custos são desnecessários. Em municípios com recursos escassos há também a possibilidade de os recursos materiais e ou tecnológicos serem insuficientes.

A hipótese de nascidos vivos serem registrados como natimortos poderia ser verificada se, no Brasil, natimortos fossem incluídos no banco de dados de nascimentos. Isso tornaria a base de dados mais confiável para comparações epidemiológicas, especialmente para avaliações de prognóstico de neonatos mais imaturos (TOMMISKA et al., 2001).

Como o indicador de BPN agrega notadamente crianças que nascem prematuramente e bebês com retardo do crescimento intra-uterino, distinguir seus componentes é crucial para se apreender as reais condições tanto da saúde materna quanto da infantil. Vários autores recomendam fazer essa distinção em razão da heterogeneidade etiológica (PEACOCK et al., 1995; KRAMER et al., 2000).

\section{RNBP a termo e pós-termo}

Em Goiás, verificou-se que a maioria dos RNBP $(55,67 \%, 2.944 / 5.288)$ era de RN a termo e pós-termo (tabela 2). Esses valores foram próximos aos dos estados da Bahia $(59,96 \%)$, Paraná $(51,54 \%)$ e Mato Grosso (55,19\%) (DATASUS, 2003a).

Nos Estados Unidos, em 1999, esses RN 
representaram $33,18 \%$ dos RNBP (VENTURA et al., 2001), indicando a ocorrência de menor proporção de retardo do crescimento intra-uterino naquele país.

Neste trabalho, a prevalência de RN pequenos para a idade gestacional não pode ser calculada em razão das variáveis contidas na Declaração de Nascido Vivo. Considerando os RNBP a termo e póstermo $(3,40 \%$, IC95\% 3,28-3,53) como RN pequenos para a idade gestacional, deve-se ter em conta que a exclusão dos RNPT e dos de gravidez múltipla subestima a prevalência de $\mathrm{RN}$ pequenos para a idade gestacional pela maior freqüência de retardo do crescimento intra-uterino entre os RNPT (ALMEIDA e MELLO JORGE, 1998; GILBERT e DANIELSEN, 2003).

Altas prevalências de BPN têm grande probabilidade de indicar que sua maioria é composta por RN pequenos para a idade gestacional (BAKKETEIG et al., 1998), mas não parece ser o caso dos recém-nascidos no estado de Goiás que, como visto anteriormente, apresenta proporções relativamente baixas de RNBP e de RNPT.

No Brasil, as investigações que incluíram prétermos nas investigações sobre pequenos para a idade gestacional obtiveram taxas variáveis de RN pequenos para a idade gestacional: $8,9 \%$ no município de Pelotas (RS) em 1993 (HORTA et al., 1997), 4,3\% em Santo André (SP) no ano de 1992 (ALMEIDA e MELLO JORGE, 1998) e $14,2 \%$ em São Luís (MA) entre os anos de 1997 e 1998 (SILVA et al., 2003).

Os dados do DATASUS (2003a), que excluem RNPT, mas que incluem os RN de gravidez múltipla, indicam $4,53 \%$ de neonatos não pré-termos e com baixo peso ao nascer na Bahia, 4,94\% em São Paulo, 4,35\% no Rio de Janeiro, 3,48\% no Ceará e 3,71\% em Goiás. É difícil pressupor se essas diferenças de proporções representam mais RN pequenos para a idade gestacional de gestações únicas ou múltiplas, porque o banco de dados disponível on-line não discrimina o tipo de gestação.

Entretanto, a probabilidade de neonatos de gravidez múltipla serem pequenos para a idade gestacional é maior porque a taxa de crescimento fetal diminui mais precocemente em virtude da influência de falta de espaço disponível no útero materno (HARRISON et al., 1971).

\section{Fatores associados ao BPN}

Neste estudo, como todas as variáveis analisadas foram associadas ao baixo peso ao nascer nas análises univariada e de regressão logística serão discutidos somente os resultados ajustados.

As faixas etárias maternas abaixo de 20 e acima de 34 anos foram fator associado ao BPN e as faixas extremas apresentaram maiores OR. Esses resultados concordam com o estudo conduzido em Salvador (BA) (SOLLA et al., 1997), mas não com os resultados de Silva et al. (1998) que relataram risco ajustado apenas para idade materna avançada.

Sobre essa questão, Strobino et al. (1995) estudaram três diferentes hipóteses para explicar o BPN de filhos de adolescentes nos Estados Unidos: desvantagem social, imaturidade biológica e comportamento não saudável durante a gestação. Nesse estudo, os resultados do modelo hierárquico de regressão apontaram o ambiente de privação social como o fator mais relevante.

Todavia, uma investigação em Utah (EUA) (FRASER et al., 1995) mostrou risco de RNBP para mães menores de 17 anos, casadas, não fumantes, com apropriados níveis de escolaridade e que receberam adequados cuidados pré-natais, quando comparadas às mães de 20 a 24 anos com as mesmas características sociodemográficas $(\mathrm{OR}=2,0$, IC95\% 1,2-3,1).

No presente estudo, mães não casadas apresentaram 1,23 vezes mais chance de BPN que as casadas. Entretanto, RNBP não foram associados a mães sem companheiro em Campinas (CARNIEL et al., 2003) e em Ribeirão Preto (SILVA et al., 1998), municípios do estado de São Paulo que se incluem entre os 25 melhores índices de desenvolvimento humano do Brasil (MARTINS, 2003).

Estudos em países desenvolvidos foram inconclusivos para indicar se o status marital é um determinante independente de BPN, RNPT e de retardo do crescimento intra-uterino. Isso porque o casamento ou co-habitação dos pais do neonato tem se tornado independente da situação educacional, ocupação e renda, refletindo mais uma escolha por um estilo de vida entre as classes sociais média e alta (KRAMER, 1987). Em Kentucky (EUA), o status marital teve efeito independente sobre o peso ao nascer, mas foi dependente de interações entre etnia, risco biológico e cuidado pré-natal (ABEL, 1997).

Em relação à escolaridade, somente as mães que não sabiam ler e escrever foram associadas ao BPN $(p=0,006)$, sugerindo apenas associação entre o BPN e as camadas sociais mais desprivilegiadas.

No entanto, uma investigação na cidade de São Paulo (SP), entre 1976 e 1998, mostrou que nos estratos socioeconômicos mais baixos, houve redução da proporção de BPN, aparentemente motivada pelo melhor crescimento intra-uterino; enquanto que nos estratos mais altos, identificou aumento da prevalência de BPN, aparentemente, pelo aumento da freqüência de recém-nascidos prematuros (MONTEIRO et al., 2000a).

Trabalhos realizados na cidade de São Paulo sobre a evolução dos fatores condicionantes da saúde na infância apontaram melhoria da renda e de escolaridade (MONTEIRO e FREITAS, 2000), das condições de moradia das crianças, do saneamento do meio, da coleta de lixo e do abastecimento de água (MONTEIRO e NAZÁRIO, 2000).

Quando se comparou mães que não realizaram nenhuma consulta com as que fizeram sete ou mais consultas de pré-natal, os resultados deste estudo foram semelhantes ao Gama et al. (2001), que usaram o mesmo padrão de referência.

A associação de RNBP aos nascimentos não hospitalares pode indicar mulheres residentes em locais com mais difícil acesso a serviços de saúde (de 35 nascimentos não hospitalares com BPN, 25 eram 
procedentes de municípios fora da região metropolitana de Goiânia - dados não mostrados).

A associação entre o sexo feminino do neonato e BPN encontrada no presente estudo poderia ser explicada por uma parcela grande de RN pequenos para a idade gestacional na população entre os RNBP. Isso porque o sexo feminino tem efeito causal importante e bem estabelecido para retardo do crescimento intra-uterino, tanto nos países desenvolvidos como naqueles em desenvolvimento (KRAMER, 1987; THOMAS et al., 2000), mas parece não ter o esse efeito para a prematuridade (KRAMER, 1987).

Embora as proporções de partos cesáreos tenham sido muito altas no estado de Goiás, essa intervenção cirúrgica foi fator protetor para RNBP, em oposição ao resultado encontrado por Silva et al. (2001) em São Luís (MA). Esses autores relataram dificuldade em discernir cesáreas mal indicadas, dentre os casos de RNBP, daquelas realizadas com indicação clínica. Eles encontraram muitos registros de cesarianas sem nenhuma indicação médica, provavelmente representando cesáreas eletivas.

\section{Limitações do estudo}

A principal limitação deste estudo está relacionada ao uso de banco de dados secundário que define as variáveis de estudo e suas formas de categorização, fugindo do escopo deste trabalho validar os dados disponíveis. Desta forma, possíveis erros e ou falhas não puderam ser corrigidos, tais como: classificação da idade gestacional, quantidade de registros (caselas) em branco ou ignorado, dificuldade na determinação da raça e do estado civil.

A idade gestacional, especialmente, pode ter dados de classificação realizada de forma não uniforme, geralmente pela data da última menstruação e ou por avaliação do ultra-sonografista e ou pelo método de Capurro. Além disso, a categorização em faixas de idade gestacional impossibilita a identificação de $\mathrm{RN}$ pequenos para a idade gestacional.

Em virtude da inexistência no banco de dados utilizado, ainda não foi possível incluir variáveis relacionadas a outros fatores de risco conhecidos na literatura (renda, resultado de gestações anteriores, presença de doenças, índice de massa corporal, uso de álcool e cigarro, peso e altura antes e durante a gestação, mês de início do atendimento pré-natal, dentre outros).

Há necessidade, também, de melhorar o preenchimento da DN, especialmente das variáveis: presença de malformações no RN, número de filhos vivos e mortos e ocupação materna.

\section{CONSIDERAÇÕES FINAIS}

Este estudo evidenciou prevalências relativamente baixas de BPN e de RNPT. Revelou, ainda, prevalência muito elevada de mães adolescentes e de partos cesáreos, fatores passíveis de intervenção num prazo relativamente curto que podem trazer resultados positivos para a saúde materna e infantil.

Os fatores associados ao BPN foram: parto prematuro, mães com baixa e avançada idade, não casadas, que fizeram menos que sete consultas de pré-natal, que não sabiam ler e escrever, que tiveram parto não hospitalar e RN do sexo feminino.

O uso de dados do Sistema de Informações sobre Nascidos Vivos mostrou-se útil e viável para identificação das populações de risco para BPN. A busca de explicações pormenorizadas dos mecanismos que permeiam as associações encontradas, desde condições e estilos de vida, disponibilidade e qualidade dos serviços de saúde até particularidades regionais devem ser objeto de investigações futuras para possibilitar o detalhamento de estratégias de redução das desigualdades decorrentes do baixo peso ao nascer.

Os resultados deste trabalho indicam a necessidade de políticas de saúde, especialmente dirigidas para a redução de cesáreas e para a melhoria da cobertura e acesso aos serviços atenção pré-natal. Acreditamos que as autoridades públicas podem oferecer melhores oportunidades para um bom começo de vida para as crianças goianas, avançando na descentralização do sistema de saúde e protegendo a mulher grávida com estratégias intersetoriais de inclusão social.

\section{REFERÊNCIAS BIBLIOGRÁFICAS}

ABEL, M. H. Low birth weight and interactions between traditional risk factors. J Genet Psychol, v.158, n.4, p.443-456. Dec, 1997.

ALEXANDER, G. R. e C. C. KORENBROT. The role of prenatal care in preventing low birth weight. Future Child, v.5, n.1, p.103-120. Spring, 1995.

ALMEIDA, A. F. e M. H. P. MELLO JORGE. O uso da técnica de "linkage" de sistemas de informação em estudos de coorte sobre mortalidade neonatal. Rev Saúde Pública, v.30, n.2, p.141-147. Abr, 1996.

ALMEIDA, M. F. e M. H. P. MELLO JORGE. Pequenos para idade gestacional: fator de risco para mortalidade neonatal. Rev Saúde Pública, v.32, n.3, p.217-224. Jun, 1998.

BAKKETEIG, L. S., et al. Current growth standards, definitions, diagnosis and classification of fetal growth retardation. Causes and consequences of intrauterine growth retardation - IDECG Workshop. Baton Rouge, USA: Eur J Clin Nutr. Nov 11-15, 1996, 1998. p.

BARROS, F. C., et al. Ethnicity and infant health in Southern Brazil. A birth cohort study. Int J Epidemiol, v.30, n.5, p.1001-1008. Oct, 2001.

BASSO, O., et al. Change in social status and risk of low birth weight in Denmark: population based cohort study. BMJ, v.315, n.7121, p.1498-1502. Dec, 1997.

BÉHAGUE, D. P., et al. Consumer demand for caesarean sections in Brazil: informed decision making, patient choice, or social inequality? A population based birth cohort study linking ethnographic and epidemiological methods. BMJ, v.324, n.20, p.942-945. Apr, 2002. 
BELIZÁN, J. M., et al. Rates and implications of caesarean sections in Latin America: ecological study. BMJ, v.319, n.7222, p.1397-1402. Nov, 1999.

BERQUÓ, E., et al. Comportamento sexual da população brasileira e percepções sobre o HIV/Aids. Centro Brasileiro de Análise e Planejamento. Ministério da Saúde - SPS-CNDST/HIVIAIDS. Brasília: Jul, p.136. 1999

BETTIOL, H., et al. Factors associated with preterm births in Southeast Brazil: a comparison of two birth cohorts born 15 years apart. Paediatr Perinat Epidemiol, v.14, n.1, p.30-38. Jan, 2000.

BLONDEL, B., et al. The impact of the increasing number of multiple births on the rates of preterm births and low birthweight: an international study. Am J Public Health, v.92, n.8, p.1323-1330. Aug, 2002.

BORTMAN, M. Factores de riesgo de bajo peso al nacer. Rev Panam Salud Publica, v.3, n.5, p.314-321. May, 1998.

BRASIL. Ministério da Justiça. Lei $n^{\circ} 6.015$, de 31 de dezembro de 1973. Dispõe sobre os registros públicos, e dá outras providências. Brasília, 1973.

BRASIL. Lei $n^{\circ}$ 9.053, de 25 de maio de 1995. Altera a redação do art. 50 da Lei $n^{\circ} 6.015$, de 31 de dezembro de 1973, que dispõe sobre os registros públicos, e dá outras providências. Brasília, 1995.

BRASIL. Ministério da Saúde. Conselho Nacional de Saúde. Resolução $n^{\circ}$ 196/1996, de 10 de outubro de 1996. Diretrizes e normas regulamentadoras da pesquisa envolvendo seres humanos. Brasília, 1996.

BRIGGS, D. Making a difference: indicators to improve children's environmental health. World Health Organization. Geneva, p.13. 2003

BROWN, A. D., et al. Sexual relations among young people in developing countries: evidence from WHO case studies. UNDP/UNFPA/WHO/World Bank Special Programme of Research. Development and Research Training in Human Reproduction. Geneva, p.50. 2001 CARNIEL, E. F., et al. A "Declaração de Nascido Vivo" como orientadora de ações de saúde em nível local. Rev Bras Saúde Matern Infant, v.3, n.2, p.165-174. Abr-Jun, 2003.

COSTA, C. E. e S. L. GOTLIEB. Estudo epidemiológico do peso ao nascer a partir da Declaração de Nascido Vivo. Rev Saúde Pública, v.32, n.4, p.328-334. Ago, 1998.

COSTA, M. C. O., et al. Indicadores materno-infantis na adolescência e juventude: sociodemográfico, prénatal, parto e nascidos-vivos. J Pediatr, v.77, n.3, p.235-242. Mai, 2001.

D'ORSI, E. e M. S. CARVALHO. Perfil de nascimentos no Município do Rio de Janeiro: uma análise espacial. Cad Saúde Pública, v.14, n.2, p.367-379. Apr, 1998.

DATASUS. Proporção de partos cesáreos [online]. Disponível

http://tabnet.datasus.gov.br/cgi/tabcgi.exe?idb2002/f08 .def Acesso em: 15 Dez 2003.

DATASUS. Nascidos vivos - Brasil. Informações de saúde [online]. Disponível http://tabnet.datasus.gov.br/cgi/deftohtm.exe?sinasc/cn v/nvuf.def. Acesso em: 10 Jan 2004.
DATASUS. Rede hospitalar do SUS - Goiás. Informações de saúde [online]. Disponível http://tabnet.datasus.gov.br/cgi/deftohtm.exe?sih/cnv/c xgo.def. Acesso em: 10 Jan 2004.

DAVID, R. J. e J. W. COLLINS, JR. Differing birth weight among infants of U.S.-born blacks, African-born blacks, and U.S.-born whites. N Engl J Med, v.337, n.17, p.1209-1214. Oct, 1997.

DEAN, A. G., et al. Epi Info. Atlanta, Georgia: Center for Disease Control and Prevention / World Health Organization 2003.

DOBSON, R. Caesarean section rate in England and Wales hits 21\%. BMJ, v.323, n.27, p.951. Oct, 2001.

EOGAN, M. A., et al. Effect of fetal sex on labour and delivery: retrospective review. BMJ, v.326, n.18, p.137. Jan, 2003.

FRASER, A. M., et al. Association of young maternal age with adverse reproductive outcomes. $N$ Engl J Med, v.332, n.17, p.1113-1118. Apr, 1995.

FUNASA. SINASC 1999 [online]. Disponível ftp://ftp.funasa.gov.br/pub/sinasc/ . Acesso em:05 Nov 2004.

GAMA, S. G., et al. Gravidez na adolescência como fator de risco para baixo peso ao nascer no Município do Rio de Janeiro, 1996 a 1998. Rev Saúde Pública, v.35, n.1, p.74-80. Fev, 2001.

GEOGOIÁS. Estado ambiental de Goiás - 2002 [online].

http://www.agenciaambiental.go.gov.br/geogoias/.

Acesso em: 07 Jan 2004.

GILBERT, W. M. e B. DANIELSEN. Pregnancy outcomes associated with intrauterine growth restriction. Am J Obstet Gynecol, v.188, n.6, p.15961599. Jun, 2003.

GOMES, J. O. e A. H. SANTO. Mortalidade infantil em município da região Centro-Oeste Paulista, Brasil, 1990 a 1992. Rev Saúde Pública, v.31, n.4, p.330-341. Ago, 1997.

GOMES, U. A., et al. Risk factors for the increasing caesarean section rate in Southeast Brazil: a comparison of two birth cohorts, 1978-1979 and 1994. Int J Epidemiol, v.28, n.4, p.687-694. Aug, 1999.

HALL, M. H. e S. BEWLEY. Maternal mortality and mode of delivery. Lancet, v.354, n.28, p.776. Aug, 1999.

HARRISON, G. A., et al. Biologia Humana - introdução à evolução, variação e crescimento humanos. São Paulo: Companhia Editora Nacional e Editora da Universidade de São Paulo, v.7. 1971. 574 p. (Ciências Puras)

HAYWOOD, J. L., et al. Estimation of outcome and restriction of interventions in neonates. Pediatrics, v.102, n.2, p.e2024. Aug, 1998.

HECK, K. E., et al. Are very low birthweight births among American Indians and Alaska Natives underregistered? Int $J$ Epidemiol, v.28, n.6, p.10961101. Dec, 1999.

HORTA, B. L., et al. Low birthweight, preterm births and intrauterine growth retardation in relation to maternal smoking. Paediatr Perinat Epidemiol, v.11, n.2, p.140-151. Apr, 1997. 
HOTIMSKY, S. N., et al. O parto como eu vejo. ou como eu o desejo? Expectativas de gestantes, usuárias do SUS, acerca do parto e da assistência obstétrica. Cad Saúde Pública, v.18, n.5, p.1303-1311. Set-Out, 2002.

IBGE. Censo demográfico 2000. Características da população e dos domicílios. Resultados do Universo. Rio de Janeiro: Instituto Brasileiro de Geografia e Estatística. 2001. 520 p.

IBGE. Censo demográfico 2000. Trabalho e rendimento. Resultados da amostra [online]. Disponível

ftp://ftp.ibge.gov.br/Censos/Censo Demografico 2000/ trabalho rendimento/UFs/. Acesso em: 10 Jan 2004.

IBGE. Pesquisa Nacional por Amostra de Domicílios 2002. Síntese de Indicadores. Instituto Brasileiro de Geografia e Estatística. Coordenação de Trabalho e Rendimento. Rio de Janeiro, p.203. 2003b

IBGE. Síntese de Indicadores Sociais - 2002. Informação demográfica e socioeconômica. Rio de Janeiro: Instituto Brasileiro de Geografia e Estatística, v.4. 2003c. 283 p. (Estudos e pesquisas)

KABIR, Z. Low birthweight: revisited. Int J Epidemiol, v.31, n.5, p.1075. Oct, 2002.

KELLEY, L. Report of Meeting: Perinatal and neonatal mortality: levels, trends, causes and risk factors. USAID's Child Health Research Project. Maternal and Neonatal Health Program. Baltimore, Maryland 10-12 May 1999: Oct, p.48. 1999. (v.3 n.1)

KILSZTAJN, S., et al. Assistência pré-natal, baixo peso e prematuridade no Estado de São Paulo, 2000. Rev Saúde Pública, v.37, n.3, p.303-310. Jun, 2003.

KOUPILOVA, I., et al. Social determinants of birthweight and length of gestation in Estonia during the transition to democracy. Int J Epidemiol, v.29, p.118-124. 2000.

KRAMER, M. S. Determinants of low birth weight: methodological assessment and meta-analysis. Bull Pan Am Health Organ, v.65, p.663-737. 1987.

KRAMER, M. S., et al. Socio-economic disparities in pregnancy outcome: why do the poor fare so poorly? Paediatr Perinat Epidemiol, v.14, n.3, p.194-210. Jul, 2000.

LANCET. Editorial: Caesarean section on the rise. Lancet, v.356, n.9243, p.1697. Nov, 2000.

MARTINS, E. Atlas de desenvolvimento humano do Brasil. Brasília, Distrito Federal: Programa das Nações Unidas para o Desenvolvimento (PNUD), Instituto de Pesquisa Econômica Aplicada (IPEA) e Fundação João Pinheiro (FJP) 2003.

MCLEOD, A. Changing patterns of teenage pregnancy: population based study of small areas. BMJ, v.323, n.7306, p.199-203. Jul, 2001.

MEHDIZADEH, A., et al. Comparison of clinical versus ultrasound estimation of fetal weight. Am J Perinatol, v.17, n.5, p.233-236. 2000.

MINISTÉRIO DA SAÚDE. Taxa bruta de natalidade [online]. Disponível http://tabnet.datasus.gov.br/cgi/idb2002/a07.htm.

Acesso em: 15 dez 2003.

MINISTÉRIO DA SAÚDE. Relatório de gestão 1998/2001 [online]. Disponível http://dtr2001.saude.gov.br/sas/relatorio/6.2\%20redes \%20assistenciais.htm\#6.2.6. Acesso em: 24 dez 2003. MONTEIRO, C. A., et al. Tendência secular do peso ao nascer na cidade de São Paulo (1976-1998). Rev Saúde Pública, v.34, n.6 Suppl, p.26-40. Dez, 2000a. MONTEIRO, C. A., et al. Evolução da assistência materno-infantil na cidade de São Paulo (1984-1996). Rev Saúde Pública, v.34, n.6 (Supl), p.19-25. Dez, 2000b.

MONTEIRO, C. A. e I. C. FREITAS. Evolução de condicionantes socioeconômicas da saúde na infância na cidade de São Paulo (1984-1996). Rev Saúde Pública, v.34, n.6 (Suppl), p.8-12. Dez, 2000.

MONTEIRO, C. A. e C. L. NAZÁRIO. Evolução de condicionantes ambientais da saúde na infância na cidade de São Paulo (1984-1996). Rev Saúde Pública, v.34, n.6 (Supl), p.13-18. 2000.

MORAIS NETO, O. L. e M. B. A. BARROS. Fatores de risco para mortalidade neonatal e pós neonatal na Região Centro-Oeste do Brasil: linkage entre bancos de dados de nascidos vivos e óbitos infantis. Cad Saúde Pública, v.16, n.2, p.477-485. Abr-Jun, 2000.

NIE, N. H., et al. Statistical Package for the Social Sciences (SPSS). Chicago, Illinois: SPSS Inc. 2001.

OLINTO, M. T. e L. W. GALVÃO. Características reprodutivas de mulheres de 15 a 49 anos: estudos comparativos e planejamento de ações. Rev Saúde Pública, v.33, n.1, p.64-72. Fev, 1999.

OPAS. El progreso en la salud de la población. Organización Panamericana de la Salud. Washington DC, EUA, p.146. 2000

OTT, W. J. Diagnosis of intrauterine growth restriction: comparison of ultrasound parameters. Am J Perinatol, v.19, n.3, p.133-137. Apr, 2002.

PANETH, N. S. The problem of low birth weight. Future Child, v.5, n.1, p.19-34. Spring, 1995.

PEACOCK, J. L., et al. Preterm delivery: effects of socioeconomic factors, psychological stress, smoking, alcohol, and caffeine. BMJ, v.311, p.531-535. 26 Aug, 1995.

PNAD. Pesquisa Nacional por Amostra de Domicílios. Demografia. População residente, por situação de domicílio e sexo, segundo os grupos de idade. [online]. Disponível

http://www.ibge.gov.br/home/estatistica/populacao/trab alhoerendimento/pnad99/destaques. Acesso em: 20/12/2002].

PORTUGAL. Natalidade por residência das mães [online]. Disponível http://www.dgsaude.pt/estat/nat mort inf/nat $m$ inf 96 -00.htm. Acesso em: 5 nov 2003].

POTTER, J. E., et al. Unwanted caesarean sections among public and private patients in Brazil: prospective study. BMJ, v.323, n.17, p.1155-1158. Nov, 2001.

PUCCINI, R. F., et al. Eqüidade na atenção pré-natal e ao parto em área da Região Metropolitana de São Paulo, 1996. Cad Saúde Pública, v.19, n.1, p.35-45. Jan-Fev, 2003.

RATTNER, D. Sobre a hipótese de estabilização das taxas de cesárea do Estado de São Paulo, Brasill. Rev Saúde Pública, v.30, n.1, p.19-33. Fev, 1996. 
RIBEIRO, E. R., et al. Comparação entre duas coortes de mães adolescentes em município do Sudeste do Brasil. Rev Saúde Pública, v.34, n.2, p.136-142. Abr, 2000.

ROBINSON, J. S., et al. Origins of fetal growth restriction. Eur J Obstet Gynecol Reprod Biol, v.92, n.1, p.13-19. Sep, 2000.

RONDÓ, P. H., et al. The influence of maternal nutritional factors on intrauterine growth retardation in Brazil. Paediatr Perinat Epidemiol, v.11, n.2, p.152166. Apr, 1997.

ROSS, N. A., et al. Relation between income inequality and mortality in Canada and in the United States: cross sectional assessment using census data and vital statistics. BMJ, v.320, n.7239, p.898-902. Apr, 2000.

ROSSO, C. F. W. Mortalidade neonatal em Goiânia: fatores prognósticos de recém-nascidos de risco. 2003. 43 p. Tese (Mestrado) Instituto de Patologia Tropical e Saúde Pública, Universidade Federal de Goiás, Goiânia.

SILVA, A. A., et al. Trends in low birth weight: a comparison of two birth cohorts separated by a 15-year interval in Ribeirao Preto, Brazil. Bull World Health Organ, v.76, n.1, p.73-84. 1998.

SILVA, A. A., et al. Infant mortality and low birth weight in cities of Northeastern and Southeastern Brazil. Rev Saúde Pública, v.37, n.6, p.693-698. Dec, 2003.

SILVA, A. A., et al. Risk factors for low birthweight in north-east Brazil: the role of caesarean section. Paediatr Perinat Epidemiol, v.15, n.3, p.257-264. Jul, 2001.

SOLLA, J. J., et al. Análisis multifactorial de los factores de riesgo de bajo peso al nacer en Salvador, Bahia. Rev Panam Salud Publica, v.2, n.1, p.1-6. Jul, 1997.

STROBINO, D. M., et al. Mechanisms for maternal age differences in birth weight. Am J Epidemiol, v.142, n.5, p.504-514. Sep, 1995.

SZOT MEZA, J. Demographic-epidemiologic transition in Chile, 1960-2001. Rev Esp Salud Publica, v.77, n.5, p.605-613. Sep-Oct, 2003.

THOMAS, P., et al. A new look at intrauterine growth and the impact of race, altitude, and gender. Pediatrics, v.106, n.2, p.e21-26. Aug, 2000.

TOMMISKA, V., et al. A national short-term follow-up study of extremely low birth weight infants born in Finland in 1996-1997. Pediatrics, v.107, n.1, p.e2-10. Jan, 2001.

UNICEF. The state of the world's children 2004. The United Nations Children's Fund. New York: Dec, p.64. 2003

VENTURA, S. J., et al. Revised birth and fertility rates for the United States, 2000 and 2001. Natl Vital Stat Rep, v.51, n.4, p.1-18. Feb, 2003.

VENTURA, S. J., et al. Births: final data for 1999. Natl Vital Stat Rep, v.49, n.1, p.1-100. Apr, 2001.

VICTORA, C. G., et al. Explaining trends in inequities: evidence from Brazilian child health studies. Lancet, v.356, n.9235, p.1093-1098. Sep, 2000.

WAGNER, M. Choosing caesarean section. Lancet, v.356, p.1677-1680. Nov, 2000. 